\title{
Les mécanismes de la conduction thermique à l'échelle moléculaire
}

\section{Mechanics of heat conduction on a molecular scale}

\author{
PAR J. YVON

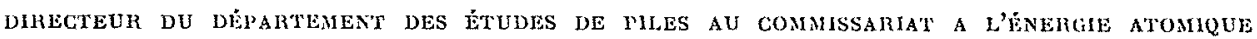

\begin{abstract}
Limitation du sujet. - Les forces intermoléculaires: Remarques qualitalives; Calcul des forces intermoléculaixes; Potentiel de Lennard Jones; Détermination des forces intermoléculaires par le second coefficient du viriel. - Les gaz hors d'équilibre: Fonction de répartition et cquation de Boltzmann; Théorie de la viscosité. Nouvelle détermination des forces intermoléculaires; Calcul de la conductibilité thermique. Nombre de Prandtl. - Remarques sur l'eau et les métaux londus.
\end{abstract}

\begin{abstract}
Limitations of the subject. - The intermolecular forces: Qualitative comments; Calculating the intermolecular forces; Lennard-Jones potential; Determining the intermolecular forces using the second birial coefficient. Gases out of equilibrium: A distribution function and Boltzmunn's equation; Viscosity theory. A new wall of determining the intermolecular forces; Calculating the thermal conductivity. P'randt's number. - Comments on water and mollen metals.
\end{abstract}

\section{LIMITATION DU SUJET}

L'exposé est limité aux fluides. Je laisserai en outre de côté les très basses températures, qui intéressent notamment l'hélium liquide et les très hautes températures - ondes de chocs, flam- mes, décharge électrique dans les gar, atmosphères stellaires.

Dans ces conditions, les phénomènes de rayonnement sont secondaires, les molécules du fluide sont des entilés indestructibles.

\section{LES FORCES INTERMOLECULAIRES}

\section{Remarques qualitatives}

Les forces intermoléculaires sont des forces répulsives aux faibles distances; à des distances plus élevées elles deviennent attractives. Leur portée est faible, elles s'annulent au-delà d'une dizaine d'angströms.

L'énergie potentielle mutuelle de deux molćcules varie en fonction de la distance, comme l'indique qualitativement la figure :

La partie attractive explique les phénomènes de cohésion, la partie répulsive explique la fai- ble compressibilité des milicux denses, liquides ou solides.

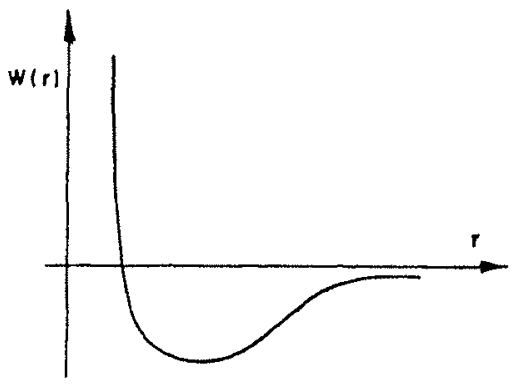


A l'exception des molécules monoatomiques, comme celles de l'hélium, des gaz rares, la représentation des forces intermoléculaires relève d'une géométrie assez complexe tandis que, dans le cas des gaz rares, chaque molécule présente la symétrie sphérique. La molécule a un centre et la mécanique intermoléculaire est une mécanique de centres de force qui exercent entre eux des actions en fonction de leur distance $r$.

L'hydrotechnique ne s'intéresse pas trop à des molécules compliquées, elle ne s'intéresse néanmoins que rarement à des molécules monoatomiques.

On traite fréquemment des molécules d'azote, d'oxygène, de gaz carbonique comme des molécules sphériques, autrement dit comme des centres de forces.

Le cas de l'eau est différent.

\section{Calcul des forces intermoléculaires}

T.e calcul des forces intermoléculaires a été effectué avec un certain soin dans le cas des atomes suivants:

hélium, néon, argon.

On doit tenir compte de la structure électronique des atomes et employer les méthodes d'approximation de la physique atomique. Le problème est lié à celui de la liaison chimique, mais la liaison chimique joue à des distances plus courtes que celles qui nous intéressent ici (le phénomène de liaison chimique n'existe pas dans le cas particulier des trois éléments cités cidessus).

Il s'agit d'un problème qui n'a pas encore été traité à fond et qui est fort difficile. Néanmoins, divers auteurs parviennent à des résultats comparables et qualitativement satisfaisants.

Un fait général est qu'aux grandes dislances, asymptotiquement, l'énergie potentielle tend vers zéro comme $1 / r^{\text {ris }}$.

\section{Potentiel de Lennard-Jones}

Soit qu'elle soit justifiée par les calculs précédents, soit qu'elle soit justifiée par ses conséquences, la formule empirique suivante, due à Lennard-Jones, est utilisée dans un grand nombre d'applications :

$$
\mathrm{W}=\varepsilon\left[\left(\frac{\sigma}{r}\right)^{1 \cdot 2}-\left(\frac{\sigma}{r}\right)^{\sigma}\right]
$$

L'exposant 6 est justifié par la théorie. Le terme qui comporte l'exposant 12 assure la répulsion aux courtes distances.

L'énergie potentielle s'annule pour :

$$
r=\sigma \text {. }
$$

Il est commode de considérer cette grandeur comme le diamètre moléculaire. Le potentiel est minimum pour :

$$
r=2^{1 / 0} \sigma .
$$

L'énergie correspondante est:

$$
-\frac{\varepsilon}{4} \text {. }
$$

En fait, dans les calculs soignés, il vaut mieux employer les résultals du calcul atomique, quand il est possible, plutôt que le potentiel empirique. Mais Je potentiel empirique permet de grouper plus de résultats. Nous nous y tiendrons ici.

\section{Détermination de $\sigma$ et $\varepsilon$ par le second coefficient du viriel}

L'équation d'état des fluides, tant que la densité reste modérée, peut se mettre sous la forme d'un développement :

$$
\frac{p \mathrm{~N}^{\top}}{\mathrm{RT}}=1+\mathrm{B} / \mathrm{N}+\mathrm{C} / \mathrm{V}^{2}+\ldots
$$

Convenons de prendre pour volume la masse moléculaire. $1, \mathrm{~B}, \mathrm{C}, \ldots$ sont des " coefficients du viriel ». B est le second coefficient du viriel. Il peut être déduit des mesures modernes sur l'équation d'état. Pour un fluide donné, c'est une fonction de la température. Mais, d'autre part, la théorie de l'équation d'état montre que:

$\mathrm{B}(\mathrm{T})=-\frac{1}{2} \mathrm{~N}_{0} \int\left[\exp \left(-\frac{\mathrm{W}(r)}{k \mathrm{~T}}-1\right)\right] 4 \pi r^{2} d r$

$\mathrm{N}_{0}$ étant le nombre d'Avogadro et $k$ le rapport $\mathrm{R} / \mathrm{N}_{6}\left(k=1,38.10^{-16} \mathrm{erg}\right.$ par degré).

L'équation (1) ne peut malheureusement pas être résolue par rapport à W. Mais elle permet d'ajuster au mieux les paramètres $\varepsilon$ et $\sigma$ du potentiel de Lennard-Jones. Quelques exemples sont donnés plus loin.

On admet et on a d'assez bonnes raisons d'admettre que ces forces, déduites d'observations sur les gaz, s'appliquent encore quand les milieux envisagés sont condensés.

\section{LES GAZ HORS D'EQUILIBRE}

La théorie en a été édifiée par Clausius, Maxwell, Boltzmann, Meyer, Enskog, Chaponan et des auteurs plus récents. 


\section{Equation de Boltzmann}

L'état d'un gaz en équilibre ou non est représenté par la fonction de répartition :

$$
\mu(x, y, z, u, v, w, t) .
$$

L'expression différentielle :

$$
\text { 2. } d x d y d z d u d v d w
$$

représente la probabilité de trouver à l'instant $t$ une molécule dans l'élément de volume:

$$
d x d y d z
$$

avec des composantes de vitesses (vitesse du centre de gravité) comprises dans l'élément:

$d u d v d w$.

La fonction de répartition évolue en fonction du temps suivant l'équation de Boltzmann :

$$
\left(\frac{\partial}{\partial t}+u \frac{\partial}{\partial x}+v \frac{\partial}{\partial y}+w \frac{\partial}{\partial z} \mu=\Delta_{c}(\mu),\right)
$$

où $\Delta_{c}(\mu)$ représente l'effet des chocs ou plus exactement des rencontres entre les molécules.

Les chocs se déroulent au hasard. $\Delta_{c}(u)$ comporte des moyennes, autrement dit des intégrales, sur les différentes possibilités qui font sortir une molécule de l'élément $d u d v d w$, ou au contraire l'y font rentrer. C'est une expression homogène du second degré par rapport à

Cette équation permet :

$1^{\circ}$ de montrer que le gaz, supposé isolé, tend vers l'équilibre thermodynamique;

$2^{\circ}$ d'édifier une théorie moléculaire de la viscosité et de la conductibilité thermique;

$3^{\circ}$ de calculer approximativement le coefficient de viscosité et le coefficient de conductibilité en fonction de la loi de force.

On doit d'abord évaluer le transfert de quantité de mouvement ou d'énergie qui s'effectue à travers un élément de surface. Le transfert, dans un gaz, est dû essentiellement au passage des molécules à travers la surface en question -dans un liquide ou un gaz comprimé il faut aussi tenir compte du transfert effectué à distance par les forces intermoléculaires, mais ce phénomène est négligeable dans un gaz. Ces évaluations conduisent aux formules suivantes :

$$
\begin{gathered}
\eta \frac{\partial \bar{\nu}}{\partial x}=m \int u v \mu d u d v d w, \\
-\lambda \frac{\partial \mathrm{T}}{\partial y}=m \int u \frac{1}{2}\left(u^{2}+v^{2}+w^{2}\right) \mu d u d v d u
\end{gathered}
$$

Tout le problème réside dans la détermination de $u$. Nous ne pouvons $\mathrm{y}$ insister ici et nous passons maintenant aux résultats. Les formules que nous donnerons ne sont qu'approchées mais suffisamment précises pour une discussion rapide.

\section{Applications à la viscosité}

Le coefficient de viscosité est donné par la formule suivante :

$$
\eta=\frac{5}{16} \frac{\sqrt{\pi m K T}}{\pi \sigma^{2} \Omega}
$$

$\Omega$ est une quantité qui dépend de la loi de force et de la température et qui est égale à l'unité lorsqu'on considère les molécules comme des billes de diamètre $\sigma$. $\Omega$ et $\pi$ sont indépendants de la densité : ce résultat n'a de sens que dans la mesure où l'équation de Bollzmann reste valable. Comme celle-ci devient incomplète lorsque des rencontres de molécules 3 à 3 deviennent fréquentes, on peut dire que la conclusion

\begin{tabular}{|c|c|c|c|c|}
\hline \multirow{2}{*}{ Gat } & \multicolumn{2}{|c|}{$\varepsilon / k \quad\left({ }^{\circ} \mathrm{K}\right)$} & \multicolumn{2}{|c|}{$\sigma \quad\left(A^{\circ}\right)$} \\
\hline & viriel & viscosite & viriel & viscosité \\
\hline $\mathrm{Ne}$ & 34,9 & 35,7 & 2,78 & 2,789 \\
\hline A & 119,8 & 124 & 3,405 & 3,418 \\
\hline $\mathrm{K}_{2}$ & 171 & 190 & 3,60 & 3,61 \\
\hline $\mathrm{Xé}$ & 221 & 229 & 4,10 & 4,055 \\
\hline $\mathrm{N}_{2}$ & 95,05 & 91,5 & 3,698 & 3,681 \\
\hline $\mathrm{O}_{2}^{2}$ & 118 & 113 & 3,46 & 3,433 \\
\hline $\mathrm{CH}_{4}$ & 148,2 & 137 & 3,817 & 3,882 \\
\hline $\mathrm{CO}_{2}^{2}$ & 189 & 190 & 4,486 & 3,996 \\
\hline
\end{tabular}
est correcte dans le domaine des gaz parfaits.

En supposant que la loi de Lennard-Jones s'applique, on peut déduire des données expérimentales, les paramètres $\varepsilon$ et $\sigma$. Le tableau suivant indique une excellente concordance avec les résultats du paragraphe précédent $\left(^{*}\right)$ :

Il est probable que les valeurs de o obtenues par l'étude de la viscosité sont meilleures que celles fournies par le second coefficient du viriel parce que la viscosité fait davantage intervenir les forces à courte distance.

De même la variation thermique de la visco-

(*) D'après Hinschrbenen, Molecular Theor!l of Gases and Liquids, N.Y. 1954. 
sité est trìs bien représentée. Voici les résultals pour le xénon :

\begin{tabular}{|c|c|c|}
\hline $\begin{array}{c}\text { Température } \\
\text { (" } \mathrm{K})\end{array}$ & $\begin{array}{c}\eta \cdot 10^{\top} \mathrm{exp.} \\
\left(\mathrm{g} \mathrm{cm}^{-1} \mathrm{sec}^{-1}\right)\end{array}$ & $\begin{array}{c}\eta \cdot 10^{-7} \\
\text { ealc. }\end{array}$ \\
\hline & 2.235 & 2.236 \\
289,7 & 2.260 & 2.260 \\
293 & 3.009 & 3.016 \\
400 & 3.351 & 3.341 \\
450 & 3.652 & 3.653 \\
500 & 3.954 & 3.951 \\
550 & & \\
\hline
\end{tabular}

Le cas de l'eau n'entre pas dans le schéma précédent parce que les molécules d'eau présentent un moment électrique - de même que, par exemple, l'oxyde de carbone ou l'ammoniac. On admet qu'il faut ajouter à l'ćnergie polentielle de Lennard-Jones l'interaction entre les moments électriques. Comme le moment électrique d'un type de molécule donné peut être déduit de la mesure de la constante diélectrique, l'ćnergie supplémentaire peut être enticrement calculée. Elle est compliquée parce qu'elle dépend de l'orientation relative des deux molécules.

La théorie du second coefficient du viriel pour ce type de molécules ne scmble pas avoir étć effectuce.

Pour la viscosité on remarque comme précédemment que les forces ont surtout de l'importance aux faibles distances : on peut admettre qu'alors les moments électriques s'orientent parallèlement. Ia force ne dépend plus que de la distance. L'énergie potentielle supplémentaire a pour expression :

$$
-\frac{2 y^{2}}{r^{3}}
$$

Cetle quantité apporte une correction assez importante.

Des ćvaluations numériques satisfaisanles ont pu alurs être effectuées pour des gaz comme $\mathrm{NH}^{3}$.

\section{Applications à la conductibilité thermique}

Quoiqu'elle puisse être améliorée, la formule (4) est excellente. A la même précision et indépendamment de la loi de force :

$$
\lambda=\frac{5}{2} c_{v} \eta
$$

Le nombre sans dimensions:

$$
\frac{i}{c_{v} \eta}
$$

\begin{tabular}{|c|c|c|}
\hline & $\lambda / c_{v} \eta$ & $\mid \begin{array}{l}\because / 4(9 \gamma-5) \\
1 / 4\end{array}$ \\
\hline Hélium & 2,44 & 2,485 \\
\hline Néon . . . . . & 2,47 & 2,44 \\
\hline Argon $\ldots \ldots \ldots$ & 2,42 & 2,51 \\
\hline Azote $\ldots \ldots \ldots \ldots$ & 1,91 & 1,91 \\
\hline Gaz carbonique .... & 1,64 & 1,70 \\
\hline Vapeur d'ean à $100^{\circ}$ & 1,24 & 1,72 \\
\hline
\end{tabular}

est donc égal à 2,5. Voici quelques valeurs (*) :

Le résultat n'est pas salisfaisant pour les gaz polyatomiques. C'est que, dans le cas, il faut tenir compte des variations de l'énergie interne des molécules et du transfert correspondant d'énergie. Sommairement disons qu'il faut ajouter l'énergie interne à l'énergie cinétique dans la formule (3). Eucken a fait un raisonnement sommaice mais ingénieux qui conduit à la relation suivante:

$$
\lambda=\frac{5}{2}\left(c_{v t}+c_{v i}\right) \eta
$$

où $c_{v /}$ représente, dans la chaleur spécifique, la part de l'énergie cinélique de translation et $c_{n i}$ la part de l'énergie interne, la diflérence des facteurs numériques provenant d'une question de degrés de liberté. Sous une forme pratique :

$$
\lambda=\frac{1}{4}\left(9 c_{p}-5 c_{v}\right) r_{1}
$$

Nous pouvons alors completer notre lableau par la valeur théorique. Les gaz polyatomiques rentrent mantenant dans la norme, sauf l'eau, et d’une manière générale les fluides polaires.

Le rapport $\lambda / c_{v} \eta$ que nous venons de calculer ressemble beaucoup à l'inverse du nombre de Prande, lequel s'écrit :

$$
\mathrm{P}_{r}=\frac{c_{p} \cdot \hat{n}}{\%}
$$

Pour un gaz monoatomique $\left(c_{p} / c_{p}\right)=\gamma=(5 / 3)$. La théorie explique donc que le nombre de Prandtl soit égal à :

$$
\frac{5}{3} \times \frac{2}{5}=0,67
$$

pour les gaz monoalomiques et n'en differe pas beaucoup pour les autres.

(*) T.H. Knevall, Kinectic theory of gases, N.Y. 1938, p. 180 . 


\section{LIQUIDES}

La théorie cinétique des liquides hors d'équilibre en est aux balbuticments. Tout juste pourrions-nous écrire les formules qui dans leur cas doivent remplacer (2) et (3).

Nous nous contenterons de faire une comparaison des nombres de Prandtl de quelques liquides caractéristiques, côte a côte avec celui des gaz monoatomiques:

$$
\begin{array}{lll}
\text { Gaz rare } \ldots \ldots \ldots \ldots & 0,67 \\
\text { Eau } \ldots \ldots \ldots \ldots & 1,7 \\
\text { Sodium fond } \ldots \ldots \ldots & 0,0075
\end{array}
$$

Le sodium liquide est un fluide remarquable : densité voisine de celle de l'eau, viscosité comparable. Ses propriétés hydrauliques sont donc très semblables à celles de l'eau. Sa chaleur spécifique, voisine de 0,3 , n'est pas très inférieure à celle de l'eau. Mais sa conductibilité thermique est très supérieure: valant le cinquième de celle de l'argent, elle est 120 fois celle de l'eau.

La conductibilité exceptionnelle du sodium liquide et aussi des autres métaux fondus est due à la présence d'électrons libres dans ces milieux. Ces électrons libres l'emportent de beaucoup sur les molécules par leur aptitude à transporter la chaleur.

Pour terminer, signalons un phénomène rócemment découvert : la conductibilití thermique des fluides deviendrait infiniment grande au voisinage du point critique (Michels, Amsterdam, 1956).

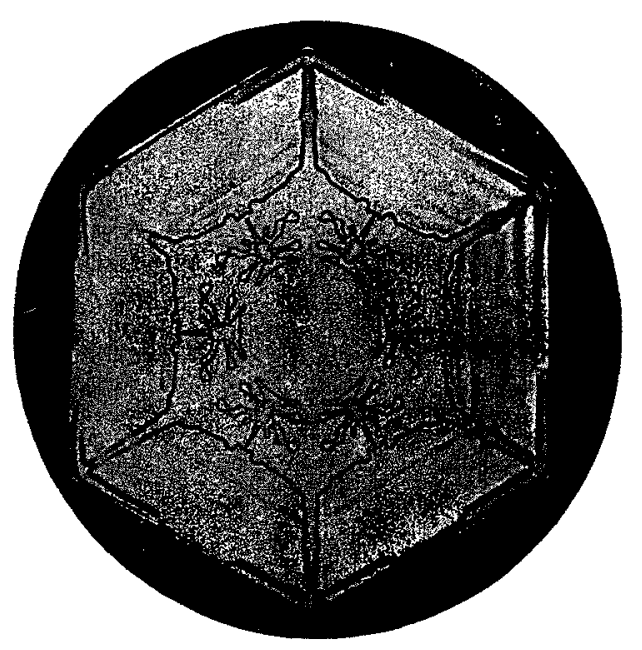

